\title{
ADVANCED-DELAY DIFFERENTIAL EQUATION FOR AEROELASTIC OSCILLATIONS IN PHYSIOLOGY
}

\author{
JORGE C. LUCERO \\ Department of Mathematics, University of Brasilia. \\ Brasilia DF 70910-900, Brazil \\ E-mail: lucero@unb.br
}

\begin{abstract}
This article analyzes a mathematical model for some aeroelastic oscillators in physiology, based on a previous representation for the vocal folds at phonation. The model characterizes the oscillation as superficial wave propagating through the tissues in the direction of the flow, and consists of a functional differential equation with advanced and delay arguments. The analysis shows that the oscillation occurs at a Hopf bifurcation, at which the energy absorbed from the flow overcomes the energy dissipated in the tissues. The bifurcation value of the flow pressure increases linearly with the tissue damping and the oscillation frequency. Also, it is minimum when the phase delay of the superficial wave to travel along the tissues is $\pi$, and increases indefinitely when the delay tends to 0 and to $2 \pi$.
\end{abstract}

Keywords: Hopf bifurcation; Lyapunov number; oscillation threshold pressure.

\section{Introduction}

Aeroelastic oscillatory phenomena appear commonly in physiology by the interaction of a flowing fluid, such as blood or air, with the surrounding elastic structure of tissues. Several of those phenomena result in the production of physiological sound. A good example is the oscillation of the vocal folds during phonation. ${ }^{1}$ Under appropriate conditions, the airflow blowing through the glottis induces their oscillation. The oscillation, in turn, modulates the airflow, which, after interacting with the oral and nasal cavities, results in the sound that we perceive as voice. This aeroelastic mechanism of sound production is common to most mammals, ${ }^{2}$ and also to songbirds by action of their syrinx's membranes. ${ }^{3}$ The same phenomenon is also responsible for the production of sound in other physiological systems, such as in blood arteries during sphygmomanometry, ${ }^{4}$ in the lips when playing a brass musical instrument,${ }^{5}$ in the nostrils when blowing the nose ${ }^{6}$ and in the soft palate when snoring. ${ }^{7}$

Almost two decades ago, Titze ${ }^{8}$ set forth the dynamical principles of the vocal fold oscillation. He proposed a mucosal wave model in which motion of the vocal fold tissues is represented as a surface wave propagating in the direction of the airflow. Since then, the original model and its several variations have been used in further studies of phonation dynamics, e.g., see Refs. 9-12, and have also been 
applied to the avian syrinx. ${ }^{13}$ The model has been particularly useful to identify the threshold conditions that its various parameters must meet in order to start the oscillation. Considering the air pressure as control parameter, the oscillation is generated through at a Hopf bifurcation of the subcritical type. This bifurcation, in combination with a cyclic fold bifurcation, produces an oscillation hysteresis phenomenon clearly visible in voicing onset-offset patterns during running speech. ${ }^{11,14}$ The bifurcation value of the air pressure, called the phonation threshold pressure, has been interpreted as a measure of ease of phonation, and proposed as a diagnostic tool for vocal health. ${ }^{15}$

A drawback of the mucosal wave model is that it assumes a small time delay for the mucosal wave to travel along the tissues. In the case of the vocal fold oscillation, the time delay is in the same order of magnitude than the period of the oscillation, ${ }^{8}$ therefore the assumption provides a rather crude approximation. In a recent work, ${ }^{16}$ an extended version of the model to the general case of arbitrary time delays for the mucosal wave has been presented. From the extended model, a theoretical equation for the phonation threshold pressure was derived, which has better qualitative agreement with experimental data than previous expression. In the present article, the extended model is considered as a general representation of aeroelastic oscillatory systems in physiology, and its dynamics is further explored.

\section{Mucosal wave model}

Let us briefly review the mucosal wave model for the vocal folds, ${ }^{8}$ shown in Figure 1. Complete right-left symmetry is assumed, and motion of tissues is allowed only in the horizontal direction. A wave propagates through the superficial tissues, in the direction of the airflow (upward).

Letting $\xi$ be displacement of the tissues from their rest position, and $y$ the vertical distance from the midpoint of the glottis in the direction of the airflow,

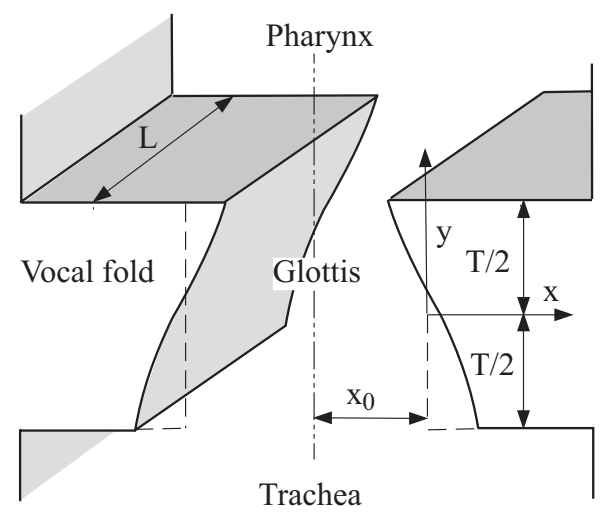

Fig. 1. Mucosal wave model of the vocal folds. 
then the tissue wave has the general expression

$$
\xi(y, t)=x(t-y / c),
$$

where $t$ is time, $x(t)=\xi(0, t)$ is the displacement of the tissues at the midpoint of the glottis and $c$ is the wave velocity.

We consider the simple case in which the vocal fold separation along the glottal height is constant, when they are at their rest position. In that case, the glottal cross sectional area $a$ at the height $y$ is $a=2 L\left(x_{0}+\xi\right)$, where $x_{0}$ is the half-width at the rest position, and $L$ is the vocal fold length. The glottal areas $a_{1}$ and $a_{2}$ at the lower $(y=-T / 2)$ and upper $(y=T / 2)$ edges of the vocal folds, respectively, are then

$$
\begin{aligned}
& a_{1}=2 L\left[x_{0}+x(t+\tau)\right], \\
& a_{2}=2 L\left[x_{0}+x(t-\tau)\right] .
\end{aligned}
$$

where $\tau=T /(2 c)$ is the time delay for the surface wave to travel half the glottal height.

The glottal aerodynamics is modeled by assuming that the subglottal pressure is constant and equal to the lung pressure $P_{L}$, and that the pressure at the exit of the glottis is the atmospheric pressure. From the lungs up to the exit of the glottis, the air flow is approximately frictionless, stationary, and incompressible. At the glottal exit, the flow detaches from the glottal wall and forms a jet stream, loosing almost all its energy by turbulence. Under such conditions, the mean glottal pressure $P_{g}$ may be expressed by

$$
P_{g}=\frac{P_{L}}{k_{t}}\left(1-\frac{a_{2}}{a_{1}}\right) .
$$

where $k_{t}$ is a transglottal pressure coefficient, and $a_{1}>0$ (open glottis).

The mechanical properties of the vocal fold tissues are lumped at the midpoint of the glottis, which yields the equation of motion

$$
M \ddot{x}+B \dot{x}+K x=P_{g},
$$

where $M, B$, and $K$ are the mass, damping, and stiffness, respectively, per unit area of the vocal fold medial surface.

Introducing Eqs. (2), (3) and (4) into Eq. (5), we obtain finally the complete equation

$$
M \ddot{x}+B \dot{x}+K x=\frac{P_{L}}{k_{t}} \frac{x(t+\tau)-x(t-\tau)}{x_{0}+x(t+\tau)}
$$

with $x_{0}+x(t+\tau)>0$.

The same model has been applied to the avian syrinx, ${ }^{13}$ and may be equally extended to other physiological systems by using appropriate values of its parameters. Note that all the systems mentioned in the introduction consists of a flow (blood or air) passing through a constricted channel (formed by an artery, the lips, 
the nostrils, or the soft palate). To generalize the above model, and at the same time reduce the number of parameters, we introduce the new adimensional variable $u=x / x_{0}$, and the parameters $\alpha=B / M, \omega=\sqrt{K / M}, p=P_{L} /\left(k_{t} x_{0} M\right)$, which yields the differential equation

$$
\ddot{u}+\alpha \dot{u}+\omega^{2} u=p \frac{u(t+\tau)-u(t-\tau)}{1+u(t+\tau)},
$$

with $1+u(t+\tau)>0$. Letting $v=\dot{u}$, the following equivalent bidimensional form is obtained

$$
\left\{\begin{array}{l}
u^{\prime}=v, \\
v^{\prime}=-\alpha v-\omega^{2} u+p \frac{u(t+\tau)-u(t-\tau)}{1+u(t+\tau)}
\end{array}\right.
$$

with $1+u(t+\tau)>0$.

\section{Small $\tau$ approximation}

Equation (8) is a functional differential equation with advance and delay arguments $(t+\tau$ and $t-\tau$, respectively). It has one fixed point (rest position) at $(u, v)=(0,0)$.

First, let us assume that the delay $\tau$ is small enough (this has been the standard assumption in previous studies, e.g., see Refs. 8-12), so that the advanced-delay terms may be approximated by the linearization

$$
u(t \pm \tau) \approx u(t) \pm \tau v(t)
$$

which reduces Eq. (8) to an ordinary differential equation

$$
\left\{\begin{array}{l}
u^{\prime}=v \\
v^{\prime}=-\alpha v-\omega^{2} u+\frac{2 p \tau v}{1+u+\tau v},
\end{array}\right.
$$

with $1+u+\tau v>0$.

The eigenvalues $\lambda$ of the Jacobian matrix at the rest position are given by the characteristic equation

$$
\lambda^{2}+(\alpha-2 p \tau) \lambda+\omega^{2}=0 .
$$

Let us consider $p$ as the control parameter, because of its direct relation with the air pressure source. The characteristic equation has a pair of conjugate complex roots, which crosses the imaginary axis from left to right as $p$ increases and crosses the bifurcation value $p_{t}=\alpha /(2 \tau)$. At this value, a Hopf bifurcation occurs, in which the rest position changes its stability: it is a stable focus for $p \leq \alpha /(2 \tau)$, and an unstable one at $p>\alpha /(2 \tau)$. At the same time, a limit cycle is generated. ${ }^{17}$

The type of Hopf bifurcation may be determined as follows: ${ }^{17}$ Let us $s$ be the signed distance along a line through the origin, and $P(s)$ be the Poincaré map for the focus. Further, let us $\sigma \equiv d^{\prime \prime \prime}(0)$ be the Lyapunov number for the focus, where $d(s)=P(s)-s$ is the displacement function. Then, if $\sigma \neq 0$, the origin is a weak focus and a Hopf bifurcation occurs at the bifurcation value of the control 
parameter, and the sign of $\sigma$ indicates its type: it is supercritical for $\sigma<0$, and subcritical for $\sigma>0$. For a general planar analytic system

$$
\begin{aligned}
& \dot{x}=a x+b y+p(x, y), \\
& \dot{y}=c x+d y+q(x, y),
\end{aligned}
$$

where $\Delta=a d-b c>0, a+d=0$, and the analytic functions $p(x, y)=$ $\sum_{i+j \geq 2} a_{i j} x^{i} y^{j}, q(x, y)=\sum_{i+j \geq 2} b_{i j} x^{i} y^{j}$, the Lyapunov number is given by

$$
\begin{aligned}
\sigma=\frac{-3 \pi}{2 b \Delta^{3 / 2}}\{ & {\left[a c\left(a_{11}^{2}+a_{11} b_{02}+a_{02} b_{11}\right)+a b\left(b_{11}^{2}+a_{20} b_{11}+a_{11} b_{02}\right)\right.} \\
+c^{2}( & \left.a_{11} a_{02}+2 a_{02} b_{02}\right)-2 a c\left(b_{02}^{2}-a_{20} a_{02}\right)-2 a b\left(a_{20}^{2}+b_{20} b_{02}\right) \\
& \left.-b^{2}\left(2 a_{20} b_{20}+b_{11} b_{20}\right)+\left(b c-2 a^{2}\right)\left(b_{11} b_{02}-a_{11} a_{20}\right)\right] \\
& \left.-\left(a^{2}+b c\right)\left[3\left(c b_{03}-b a_{30}\right)+2 a\left(a_{21}+b_{12}\right)+\left(c a_{12}-b b_{21}\right)\right]\right\} .
\end{aligned}
$$

In our case, for $p=\alpha /(2 \tau)$, we have

$$
\left\{\begin{array}{l}
u^{\prime}=v \\
v^{\prime}=-\omega^{2} u-\alpha v u-\alpha \tau v^{2}+\alpha u^{2} v+2 \alpha \tau u v^{2}+\alpha \tau^{2} v^{3}+\ldots
\end{array}\right.
$$

which produces

$$
\sigma=\frac{3 \pi \alpha}{2 \omega}\left(1+\alpha \tau+3 \omega^{2} \tau^{2}\right)>0 .
$$

and therefore the bifurcation is subcritical.

\section{General case for arbitrary $\tau$}

Let us consider now the general case, given by Eq. (8). Linearization around the equilibrium position yields

$$
\left\{\begin{array}{l}
u^{\prime}=v \\
v^{\prime}=-\alpha v-\omega^{2} u+p[u(t+\tau)-u(t-\tau)],
\end{array}\right.
$$

whose characteristic equation is

$$
\lambda^{2}+\alpha \lambda+\omega^{2}-2 p \sinh (\lambda \tau)=0
$$

For $p=0$, Eq. (17) has the roots

$$
\lambda=-(\alpha / 2) \pm \sqrt{(\alpha / 2)^{2}-\omega^{2}},
$$

which have negative real parts.

For $p>0$, Eq. (17) may have an indefinite number of roots. Let us assume a pair of imaginary roots $\lambda= \pm i \mu$. Substituting into Eq. (17), using the identity $\sinh (i x)=i \sin (x)$, and separating real and imaginary parts, we obtain

$$
\begin{aligned}
& -\mu^{2}+\omega^{2}=0 \\
& \mu \alpha-2 p \sin (\mu \tau)=0
\end{aligned}
$$


The first equation produces the oscillation angular frequency $\mu=\omega$. The value of $p$ given by the second equation is the bifurcation pressure

$$
p_{t}=\frac{\alpha \omega}{2 \sin (\omega \tau)}
$$

with $0<\omega \tau<\pi$.

According to Rouch's Theorem,${ }^{18}$ the roots of the characteristic equation depend continuously on the parameter $p$. Hence, for $0 \leq p<p_{t}$, all roots have negative real parts, and at $p=p_{t}$, a pair of roots become imaginary. We verify next that those roots cross the imaginary axis from left to right. Implicit differentiation of Eq. (17) produces

$$
[2 \lambda+\alpha-2 p \tau \cosh (\lambda \tau)] \frac{d \lambda}{d p}=2 \sinh (\lambda \tau)
$$

Substituting $\lambda=\omega i, p=p_{t}$, given by Eq. (21), and separating the real part we obtain finally

$$
\left.\frac{d \operatorname{Re}(\lambda)}{d p}\right|_{p=p_{t}}=\frac{4 \omega \sin (\omega \tau)}{\left\{\alpha^{2}[1-\omega \tau \cot (\omega \tau)]^{2}+4 \omega^{2}\right\}}>0
$$

for $0<\omega \tau<\pi$. This is the transversatility condition, which proves that the roots cross the imaginary axis and therefore their real parts become positive.

The above results imply that the equilibrium position at $x=0$ is stable for $p<p_{t}$, and unstable for $p>p_{t}$. Further, by the Hopf Bifurcation Theorem for functional differential equations, ${ }^{19}$ a limit cycle is generated at $p=p_{t}$.

\section{Conditions for the oscillation onset}

The oscillation threshold pressure is then given by Eq. (21). Let us $\delta=2 \omega \tau$ denote the phase delay for the surface wave to travel the whole constricted channel (recall that $\tau$ is the time delay to travel half that distance). We may rewrite the above equation as

$$
p_{t}=\frac{\alpha \omega}{2 \sin (\delta / 2)}, \quad 0<\delta<2 \pi
$$

This equation tells us that the oscillation threshold pressure increases with the tissue damping $\alpha$ and the oscillation frequency $\omega$, and depends on the phase delay $\delta$ following a cosecant characteristic, as shown in Fig. 2. Its minimum value occurs at $\delta=\pi$. Note also that, for $\tau \rightarrow 0, \sin (\omega \tau) \rightarrow \omega \tau$, and $p_{t} \rightarrow \alpha /(2 \tau)$, as found in the previous section.

It is instructive to consider also the exchange of energy between the airflow and the tissues at the oscillation onset, as in Ref. 11.

The energy dissipated in the tissues is the work done by the damping force

$$
W_{\alpha}=\oint_{\text {cycle }} \alpha v d x=\int_{0}^{2 \pi / \omega} \alpha v^{2} d t
$$




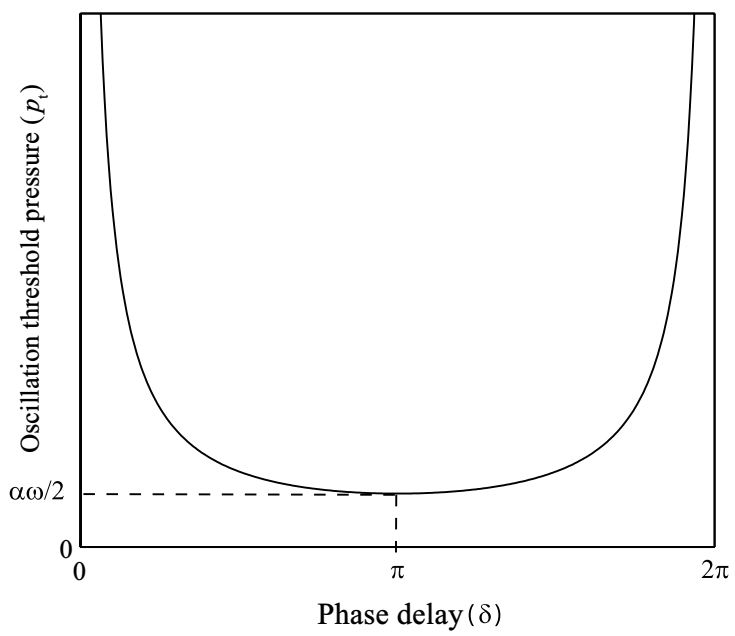

Fig. 2. Oscillation threshold pressure vs. phase delay of the surface wave to travel along the tissues.

In the vicinity of the bifurcation point, the oscillation may be described by the sinusoid $x=A \sin (\omega t)$, which produces

$$
W_{\alpha}=\pi A^{2} \alpha \omega
$$

The energy absorbed from the airflow is the work done by the air pressure

$$
W_{p}=\oint_{\text {cycle }} p_{g} d x=\int_{0}^{2 \pi / \omega} p_{g}(t) v d t
$$

where $p_{g}$ is the right side of Eq. (7). In the vicinity of the bifurcation point, $p_{g}$ may be approximated by its linear part, as done in Eq. (16), which, together with a sinusoidal approximation for $x(t)$, produces

$$
W_{p}=2 \pi A^{2} p \sin (\delta / 2)
$$

for $0<\delta<2 \pi$. Letting $W_{\alpha}=W_{p}$, we obtain the oscillation threshold condition given by Eq. (21). For $p>p_{t}$, we have $W_{p}>W_{\alpha}$ (see Figure 3 ).

We may therefore say that the oscillation is fueled by a transfer of energy from the airflow to the tissues. When the air pressure increases and passes through the oscillation threshold value, the energy absorbed from the airflow is large enough to overcome the energy dissipated in the tissues, and so an oscillation of growing amplitude may start. The transfer of energy depends on the phase delay $\delta$, following a sinusoidal characteristics. Its has a maximum at $\delta=\pi$, which is the same condition for a minimum at the threshold pressure $p_{t}$.

\section{Conclusion}

The above analysis has explored the dynamics of aeroelastic oscillations of some physiological systems. The results show that the oscillation appears at a Hopf 


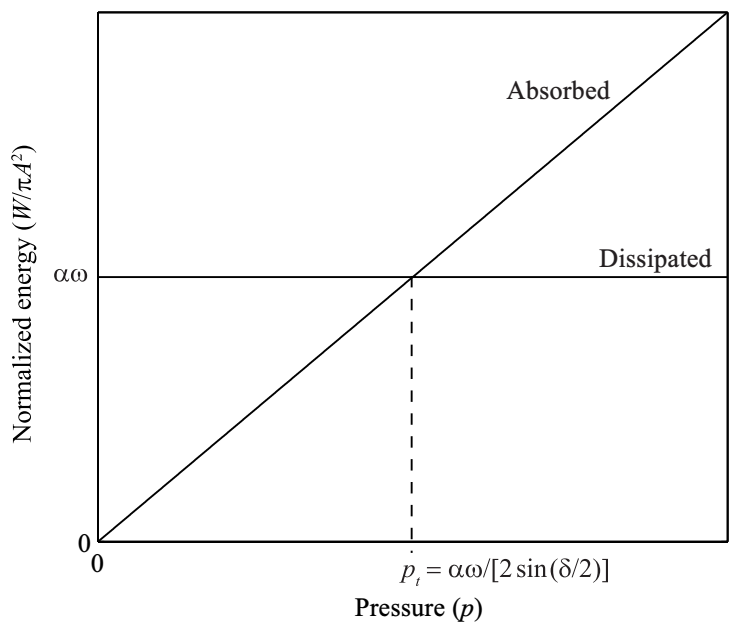

Fig. 3. Normalized absorbed and dissipated energy vs. flow pressure.

bifurcation, where the rest position of the tissues becomes unstable and a limit cycle appears. However, identification of the type of the bifurcation has been left. In the case of small time delay, the bifurcation is of the subcritical type and causes an oscillation hysteresis phenomenon. Is it still subcritical at large time delays? This should be an interesting question for further research.

\section{Acknowledgments}

This work was supported by MCT/CNPq (Brazil).

\section{References}

1. I. R. Titze, Principles of Voice Production (Prentice-Hall, Englewood Cliffs, 1994).

2. I. Wilden, H. Herzel, G. Peters and G. Tembrock, Bioacoustics 9, 171 (1998).

3. F. Goller and O. N. Larsen, P. Natl. Acad. Sci. USA 94, 1487 (1997).

4. J. B. Grotberg and O. E. Jensen, Annu. Rev. Fluid Mech. 36, 121 (2004).

5. S. Adachi and M. A. Sato, J. Acoust. Soc. Am. 99, 1200 (1996).

6. D. H. Hodges, G. A. Pierce, O. A. Bauchau and M. J. Smith, AE 6200: Aeroelasticity - Class Notes (Georgia Institute of Technology, Atlanta, 2006).

7. Y. Aurgan and C. Depoiller, J. Sound Vib. 188, 39 (1995).

8. I. R. Titze, J. Acoust. Soc. Am. 83, 1536 (1988).

9. R. W. Chan and I. R.Titze, J. Acoust. Soc. Am. 119, 2351 (2006).

10. C. Drioli, J. Acoust. Soc. Am. 117, 3184 (2005).

11. J. C. Lucero, J. Acoust. Soc. Am. 105, 423 (1999).

12. J. C. Lucero, Comm. Math. Sci. 3, 517 (2005).

13. R. Laje and G. B. Mindlin, Phys. Rev. E 72, 036218 (2005).

14. L. L. Koenig, W. E. Mencl, and J. C. Lucero, J. Acoust. Soc. Am. 118, 2535 (2005).

15. I. R. Titze, S. S. Schmidt, and M. R. Titze, J. Acoust. Soc. Am. 97, 3080 (1995).

16. J. C. Lucero and L. L. Koenig, J. Acoust. Soc. Am. 121, 3280 (2007). 
17. L. Perko, Differential Equations and Dynamical Systema, (Springer-Verlag, New York, 1991).

18. J. Dieudonn, Foundations of Modern Analysis (Academic Press, New York, 1960).

19. J. Hale, Theory of Functional Differential Equations (Springer-Verlag, New York, 1977). 